\title{
SIMULATION OF ERROR TRAPPING DECODERS ON A FADING CHANNEL
}

\author{
G. Sharma ${ }^{\ddagger}$, A. Dholakia*, and A. Hassan ${ }^{\dagger}$ \\ ‡ ECE Dept., North Carolina State University, Raleigh, NC 27695- 7911 \\ * IBM Corp., 3039 Cornwallis Rd., Research Triangle Park, NC 27709 \\ ${ }^{\dagger}$ Ericsson Inc., 1 Triangle Drive, Research Triangle Park, NC 27709
}

\begin{abstract}
In this paper, we evaluate the performance of error trapping decoding when used to protect data over a mobile radio channel. The channel is represented by the Gilbert-Elliott(GE) model, with its parameters related to the physical quantities determining the fading statistics. Simulations are used to evaluate codeword and bit error probabilities for three different error correcting codes for a number of scenarios. Using simplying assumptions, analytical expressions are derived for the codeword error probabilities and these are shown to be in excellent agreement with simulation results.
\end{abstract}

\section{INTRODUCTION}

The received signal envelope in a mobile digital cellular system is known to display Rayleigh statistics. This Rayleigh fading is characterized in the digital domain by a channel having burst errors. The Gilbert-Elliott model $[3,4]$ provides a useful discrete model for such a channel where the parameters of the model can be readily related to the statistics of the fade $[1,6]$. The simplicity of the model makes it attractive for analyzing the performance of error control codes through simulations or through exact/approximate analysis. Analytical expressions for error probabilities were first derived in $[3,4]$ and more recently in $[8,9]$ for a simplified versions of the GE model. However, all the analytical expressions were aimed at determining codeword error performance for random error correcting codes and bit error statistics and burst error correcting codes were not considered. In this paper, we consider the performance of three binary codes: the $(23,12)$ Golay Code and a $(14,6)$ cyclic burst error correcting code (BECC) with burst correcting capability (BCC) of 4 , and a $(23,13)$ shortened cyclic code with BCC of 5 . The performance of the three codes on a Gilbert-Elliott channel are compared through simulations for BPSK and DPSK modulation schemes. We also present a simpli-

\footnotetext{
*This research was performed while A. Dho lakia was at NCSU.
}

fied analysis for a restricted range of model parameters and compare the theoretical results with simulations.

\section{CHANNEL MODEL}

The Gilbert-Elliott(GE) model [3, 4] was chosen to model the fading channel in the simulations. In the model, the channel is a binary symmetric channel (BSC) with memory determined by a two state Markov chain. This model is shown schematically in Fig. 1. A channel has two states, a good $(\mathrm{G})$ state and a bad $(\mathrm{B})$ state with transition probabilities $\alpha$ and $\beta$ as shown in the figure. In either state the channel is represented as a binary symmetric channel (BSC) with the probability of bit error given by $p_{g}$ in the good state and $p_{b}$ in the bad state.


Figure 1: The Gilbert-Elliott Channel Model.

Assuming that the channel fades slowly with respect to a bit interval the parameters of the model can be related to various physical quantities. To obtain such a relation, note that Rayleigh fading results in an exponentially distributed multiplicative distortion of the signal. As a result, the probability density function of the signal-to-noise ratio (SNR), $\lambda$, is given by $[1,6]$

$$
f(\lambda)=\frac{1}{\lambda_{0}} \exp \left(-\frac{\lambda}{\lambda_{0}}\right) \quad \lambda>0
$$


where $\lambda_{0}$ is the average SNR.

The channel is said to be in the $\operatorname{good}(G)$ state while the SNR is above a threshold $\lambda_{T}$ and once the SNR falls below $\lambda_{T}$ the channel goes into the bad(B) state. Using the level crossing rate and the SNR density function, the transition probabilities can be found in terms of physical quantities as follows $[1,6]$ :

$$
\begin{aligned}
\alpha & =\frac{f_{d} T \sqrt{2 \pi \Gamma}}{\exp (\Gamma)-1} \\
\beta & =f_{d} T \sqrt{2 \pi \Gamma}
\end{aligned}
$$

where $T$ is the symbol interval, $\Gamma=\frac{\lambda_{T}}{\lambda_{0}}$ and $f_{d}$ is the maximum Doppler speed given by

$$
f_{d}=\frac{v f_{c}}{c},
$$

where $v$ is the vehicle speed, $f_{c}$ is the carrier frequency, and $c$ is the speed of light $\left(3 \times 10^{8} \mathrm{~m} / \mathrm{s}\right)$. The symbol interval is usually specified in terms of the symbol rate $R_{s}=\frac{1}{T}$.

The bit error rates in each state are determined for a given modulation scheme by appropriately averaging the BER for an AWGN expressed as a function of SNR. Thus if $E(\lambda)$ is the BER for an Additive White Gaussian Noise (AWGN) channel with SNR of $\lambda$ for the given modulation scheme then,

$$
\begin{aligned}
& p_{g}=\frac{\int_{\lambda_{T}}^{\infty} E(\lambda) f(\lambda) d \lambda}{\int_{\lambda_{T}}^{\infty} f(\lambda) d \lambda} \\
& p_{b}=\frac{\int_{0}^{\lambda_{T}} E(\lambda) f(\lambda) d \lambda}{\int_{0}^{\lambda_{T}} f(\lambda) d \lambda}
\end{aligned}
$$

We shall concentrate on two modulation schemes BPSK and DPSK. Simplified expressions for $p_{b}$ and $p_{g}$ for these modulation schemes are provided in [6].

\section{SIMULATION METHODOLOGY}

Three linear block codes were chosen for performance evaluation ${ }^{1}$. The first is the cyclic Golay $(23,12)$ code with a minimum distance of 7 which has the generator polynomial, $g_{1}(x)=x^{11}+x^{10}+x^{6}+x^{5}+x^{4}+x^{2}+1$. The other two codes chosen were burst error correcting codes with redundancy and block length similar to the Golay code. The second code is a cyclic $(14,6)$ burst error correcting code from [2, pp. 152] capable of correcting all error bursts having burst length 4 or less. The generator polynomial for this code is $g_{2}(x)=x^{8}+x^{6}+x^{4}+1$. The third code chosen for study

\footnotetext{
${ }^{1}$ Note that for the study of linear block codes an iid bit-stream generator is unnecessary for simulations.
}

was a $(23,13)$ code obtained by shortening a $(341,331)$ cyclic burst error correcting code with burst correcting capability of 5 , having the generator polynomial $g_{3}(x)=x^{10}+x^{8}+x^{7}+x^{5}+x^{4}+x^{3}+1$.

Systematic encoding was used for all codes. Decoding for the Golay code was performed using the Meggitt decoder obtained by modifying an error trapping decoder [2]. Since the Golay code is a perfect code this corresponds to complete decoding and hence there were no uncorrectable error events detected. For the burst error correcting codes error trapping decoders were used. For the $(14,6)$ cyclic burst error correcting code (BECC) the error trapping decoder corrects all bursts of cyclic length 4 or less. Since the shortened $(23,13)$ code is not cyclic, it is capable of correcting only non-cyclic bursts with burst length 5 or less. For both the burst error correcting codes, the error trapping decoder performs incomplete decoding and there are error events detected that cannot be corrected. In these cases, the codeword was declared to be in error and the information bits were passed on for computation of bit error statistics without any correction.

\section{SIMPLIFIED ANALYSIS}

For typical vehicle velocities and symbol rates for American Digital Cellular and GSM, it can be seen that the channel parameters $\alpha$ and $\beta$ in (2) and (3) are extremely small in relation to the inverse of typical codeword block lengths. As a result, for these scenarios the transitions between states are extremely infrequent, and one may assume that for the duration of a codeword the channel remains in the state in which the codeword began. With this approximation, the probability of codeword error on the GE channel is readily obtained as

$$
P_{w}=\pi_{G} P_{c}\left(p_{g}\right)+\pi_{B} P_{c}\left(p_{b}\right)
$$

where $\pi_{G}=\alpha /(\alpha+\beta)$ and $\pi_{B}=\beta /(\alpha+\beta)$ are the steady state probabilities of being in the good and bad state, respectively, and $P_{c}(p)$ represents the probability of codeword error on a BSC with symbol error probability $p$.

For an $(\mathrm{n}, \mathrm{k})$ random error correcting code capable of correcting $t$ random errors,

$$
P_{c}(p) \equiv P_{c}^{R E C}(n, t, p)=1-\sum_{i=0}^{t}\left(\begin{array}{c}
n \\
i
\end{array}\right)(1-p)^{n-i} p^{i} .
$$

The corresponding expression for a BECC capable of correcting all cyclic bursts with burst length $(\mathrm{BL}) \leq l$ is obtained from,

$$
P_{c}(p) \equiv P_{c}^{C B E C}(n, l, p)
$$




$$
\begin{aligned}
= & 1-\operatorname{Prob}(\text { No error })- \\
& \sum_{i=1}^{l} \operatorname{Prob}(\mathrm{BL}<l \text { and i errors }) .
\end{aligned}
$$

Since on a BSC all error patterns with $i$ errors are equiprobable

$$
\operatorname{Prob}(\mathrm{BL}<l \mid \text { i errors })=\frac{n\left(\begin{array}{c}
(l-1) \\
(i-1)
\end{array}\right)}{\left(\begin{array}{c}
n \\
i
\end{array}\right)},
$$

from which it follows that,

$$
\begin{aligned}
P_{c}^{C B E C}(n, l, p)= & 1-(1-p)^{n}- \\
& \sum_{i=1}^{l} n\left(\begin{array}{c}
(l-1) \\
(i-1)
\end{array}\right)(1-p)^{n-i} p^{i} .
\end{aligned}
$$

In a similar fashion, it can be shown that for a noncyclic BEC with BCC of $l$,

$$
\begin{aligned}
P_{c}(p) \equiv & P_{c}^{B E C}(n, l, p) \\
= & 1-(1-p)^{n}- \\
& \sum_{i=1}^{l}(n+1-l)\left(\begin{array}{c}
(l-1) \\
(i-1)
\end{array}\right)(1-p)^{n-i} p^{i}- \\
& \sum_{i=1}^{l} \sum_{r=1}^{l-i}\left(\begin{array}{c}
(l-1-r) \\
(i-1)
\end{array}\right)(1-p)^{n-i} p^{i} .
\end{aligned}
$$

\section{SIMULATION RESULTS}

Using the channel model, Monte-Carlo simulations were performed. The threshold $\Gamma$ was set to 0.1 so that an SNR $10 \mathrm{~dB}$ below the average SNR represents the transition to the bad state. In order to represent a wide range of mobile communication environments, the product $f_{d} T$ was considered as an independent parameter and simulations were performed for $f_{d} T=10^{-3}$, $0.01,0.05$, and 0.1 . These values encompass the whole gamut of mobile communication environments ranging from cellular telephony to PCS. Two modulation schemes, BPSK and DPSK, were considered in the simulations. For each combination of these parameters simulations were performed for an average SNR varying from $0 \mathrm{~dB}$ to $26 \mathrm{~dB}$ in steps of $2 \mathrm{~dB}$ and codeword and bit error rates were estimated. For extremely small values of $\alpha$ and $\beta$ channel transitions may become extremely rare events, hence the simulations determined the codeword error and bit error probabilities for codewords beginning in a given state. These were then weighted by the steady state probability of the corresponding state and added to obtain the overall codeword and bit error probabilities.

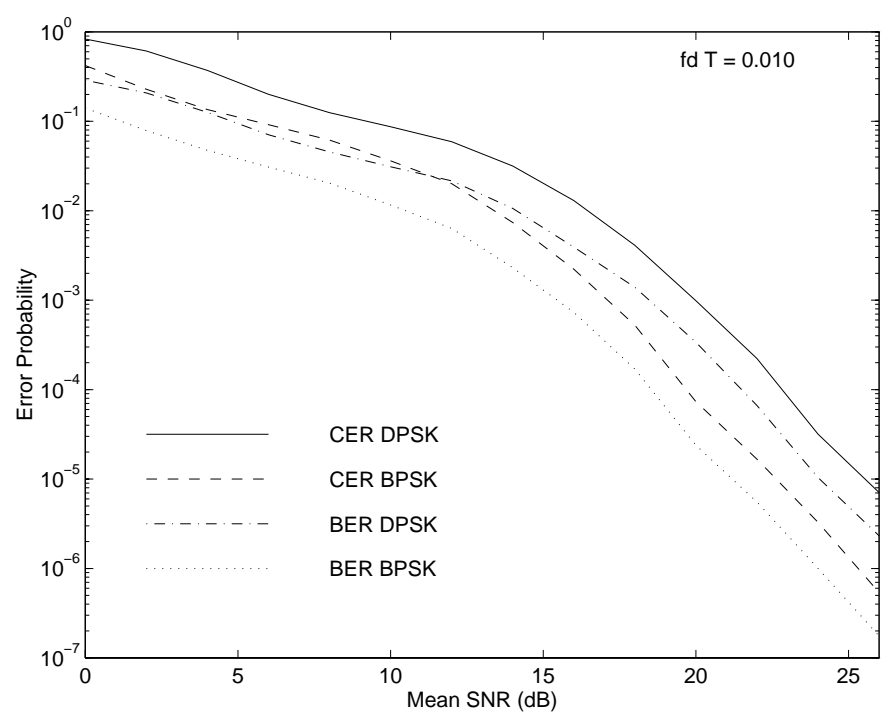

Figure 2: CER and BER estimates for the Golay code with BPSK and DPSK.

We first consider the difference in performance of the BPSK and DPSK modulation schemes. Figure 2 contains a plot of the average SNR vs. estimated bit error rate (BER) and codeword error rate (CER) for the Golay code for $f_{d} T=0.01$, for BPSK and DPSK modulation schemes. From the graphs one can see that over the moderate and high SNR region BPSK is $3 d B$ better than DPSK. This result is in agreement with the uncoded bit error probabilities for BPSK and DPSK on a fading channel [7, pp. 718]. The results for other codes and parameter values have similar characteristics. Hence, in the subsequent discussion results are presented only for BPSK modulation. One can also see from the plots that the codeword and bit error rates show identical trends and hence we shall use only the BER estimates in most of the following discussion.

Next we consider the impact of the parameter $f_{d} T$ on each of the codes. Figure 3 contains plots of the estimated bit error rate (BER) vs. average SNR for the Golay code with BPSK modulation, for $f_{d} T=10^{-3}$, $0.01,0.05$, and 0.1 and Fig. 4 and 5 contain the corresponding plots for the $(14,6)$ cyclic burst error correcting code and the $(23,13)$ burst error correcting code, respectively. From the figures, it is clear that an increase in $f_{d} T$ improves the performance of the Golay code significantly whereas it leads to only marginal improvement in the performance of the two burst error correcting codes. These results agree with the intuition that the errors tend to be more random as the channel parameters $\alpha$ and $\beta$ increase. Since the Golay 


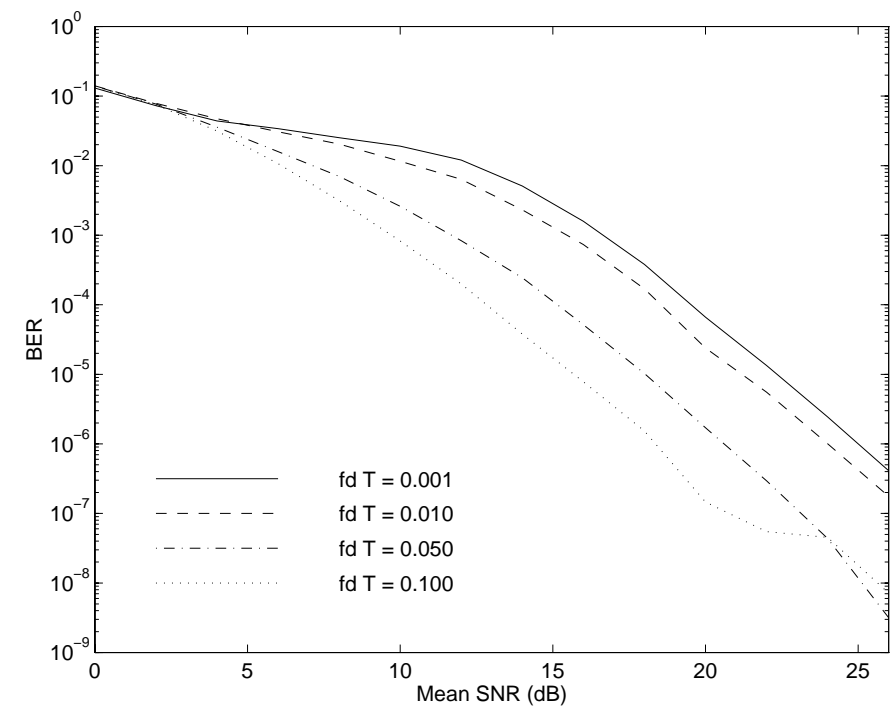

Figure 3: BER for the Golay code with BPSK.

code is capable of correcting random errors it benefits significantly from the increase in randomness whereas the burst error correcting codes make only marginal gains.

We now compare the performance of the different codes. Figure 6 contains a plot of the BER vs. average SNR for the three codes for BPSK modulation and $f_{d} T=0.01$. From the plots one can see that for these parameter values the performance of the three codes is similar for values of the average SNR below $12 \mathrm{~dB}$. Above $12 \mathrm{~dB}$ the performance of the two burst error correcting codes is similar while that of the Golay code is significantly better. For the channel model that we are considering, at high SNR's the probability of error in both the good state and the bad state decreases. As a consequence, the errors at high SNR's are less correlated. This works once again to the advantage of the Golay code capable of correcting random errors, whereas the burst error correcting codes only make marginal gains due to the decreased number of symbol errors. Since bursts of length 4 and 5 will rarely have more than 3 symbol errors the Golay code performs better or as well as the burst error correcting codes in most scenarios. In this regard, it is worth noting that a burst error correcting code will give optimum performance over a channel producing bursts close to its burst correcting capability, whereas a random error correcting code is more robust to channel parameter variations.

Finally, we investigate the validity and accuracy of the simplified expressions for codeword error rates derived in section 4 . Figure 7 contains plots of compar-

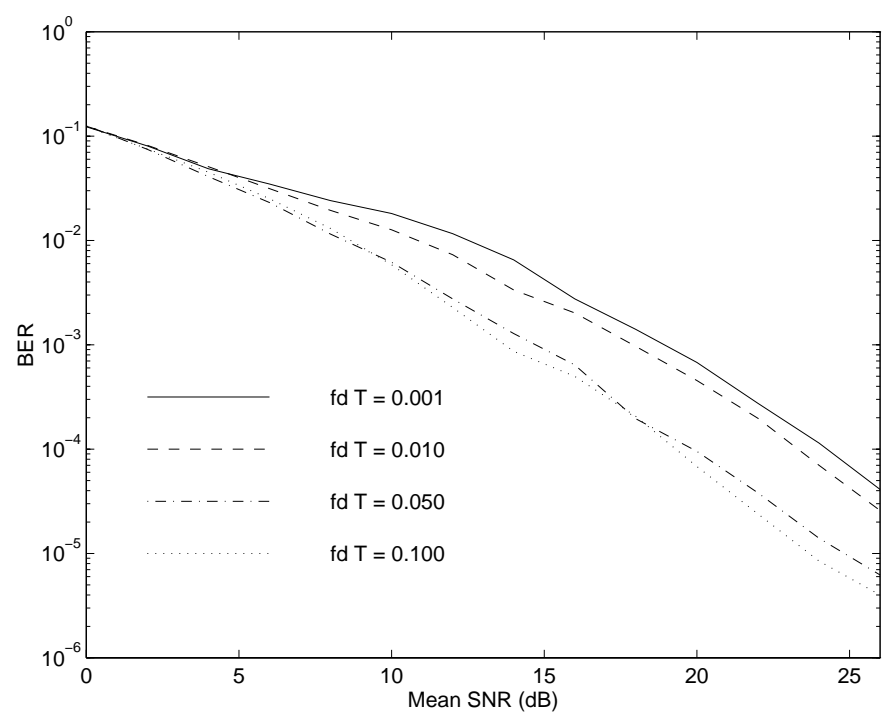

Figure 4: BER for the $(14,6)$ BECC code with BPSK.

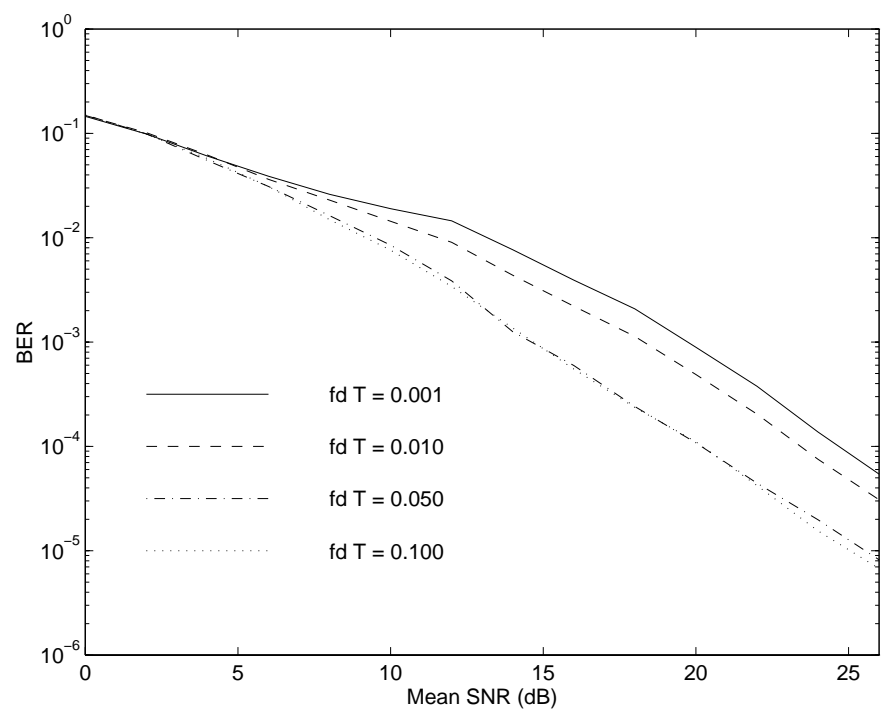

Figure 5: BER for the $(23,13)$ BECC code with BPSK. 


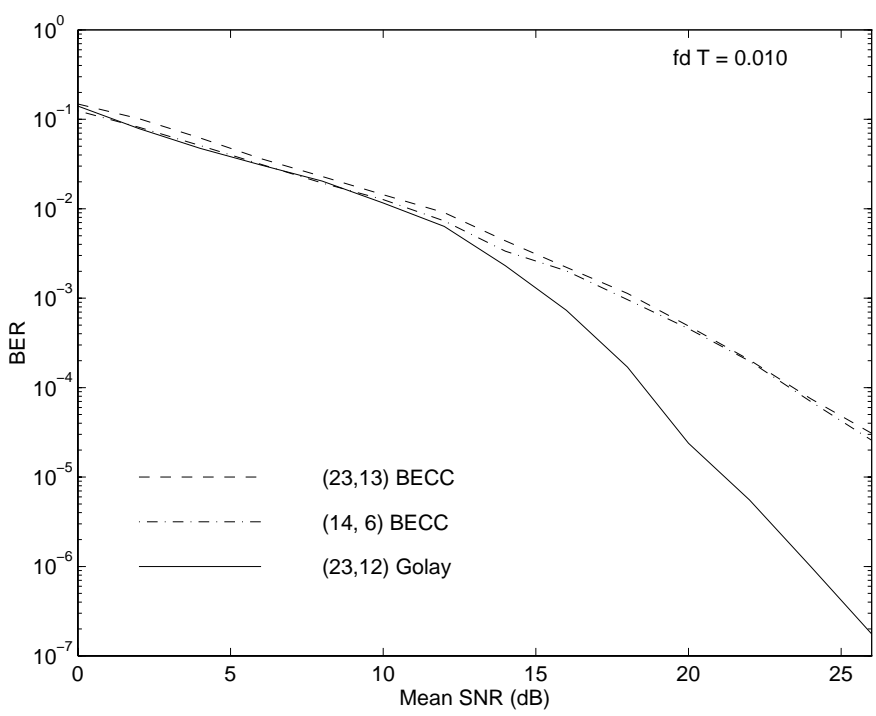

Figure 6: BER for the three codes with BPSK and $f_{d} T=0.01$.

isons of simulations and the simplified theoretical expressions for the three codes with BPSK modulation and for $f_{d} T=0.001$. For this case, $\alpha \approx 0.0754$ and $\beta \approx 0.00079$ and hence the assumption that the channel rarely changes state in the middle of a codeword is valid and therefore the theoretical results agree extremely well with the simulations. However, for higher values of $f_{d} T=0.01$ the assumption is no longer valid and hence the simulation results deviate significantly from the simplified theoretical expressions.

\section{CONCLUSIONS}

In this paper, we simulated the performance of three linear block codes on a fading channel modeled by the Gilbert-Elliott model and considered the impact of several parameters on the performance of the codes. The relative performance for different modulation schemes was seen to be in agreement with their uncoded bit error rates. For the parameters considered the random error correcting Golay code performed better than the burst error correcting codes. For some channel parameter values of our interest, analytical expressions for codeword error probability were derived and found to be in excellent agreement with the simulations.

\section{REFERENCES}

[1] L. Ahlin, "Coding Methods for the mobile radio channel," Nordic Seminar on Digital Land Mobile Communication, Feb. 1985, Espoo, Finland.



Figure 7: Comparison of theoretical CER estimates with simulations.

[2] R.E. Blahut, Theory and Practice of Error Control Codes, Addison-Wesley, Menlo Park, California, 1983.

[3] E.N. Gilbert, "Capacity of a burst noise channel," Bell Syst. Tech. J., vol. 39, pp. 1253-1266, Sept. 1960.

[4] E.O. Elliott, "Estimates of error rates for codes on burst-noise channels," Bell Syst. Tech. J., vol. 42, pp. 1977-1997, Sept. 1963.

[5] S. Lin and D.J. Cosetllo,Jr., Error Control Coding: Fundamentals and Applications, Prentice Hall, NJ, 1983.

[6] R. Krishnamurthi, An Analytical Study of Block Codes in a Portable Digital Cellular System, Ph.D. Thesis, SMU, 1990.

[7] J.G. Proakis, Digital Communications, 2nd ed., McGraw Hill, 1989.

[8] B. Wong and C. Leung, "On computing undetected error probabilities on the Gilbert channel," IEEE Trans. Comm., vol. 43, no. 11, pp. 26572661, Nov. 1995.

[9] J.R. Yee and E.J. Weldon, Jr., "Evaluation of the performance of error correcting codes on a Gilbert channel," IEEE Trans. Comm., vol. 43, no. 8, pp. 2316-2323, Aug. 1995. 